\title{
Performance of Filtered OFDM Over Different Fading Channels
}

\author{
Asia Hazareena, B. Aziz Musthafa
}

\begin{abstract}
Orthogonal frequency division multiplexing (OFDM) schemes are used extensively nowadays in large number of practical systems, but OFDM may not be the ideal solution for $5 G$ scenarios because OFDM has high Out of band emission (OOBE) due to high spectral leakage and high peak to average power ratio (PAPR). Therefore Filtered OFDM (F-OFDM), which uses sub band filtering, is proposed as the most flexible waveform for $5 G$. However there is no work listed in the literature so far that compares the bit error rate (BER) performance of F-OFDM and conventional OFDM in AWGN, Rayleigh and Rician channel. This paper presents the simulation results on the $B E R$ performance of F-OFDM and OFDM in presence of AWGN, Rayleigh and Rician channel. The comparison is also done in terms of OOBE and PAPR factor. Results indicate that F-OFDM shows significantly less spectral leakage with same performance in terms of BER compared to conventional OFDM. However for the cases considered, OFDM performance is slightly better than F-OFDM in terms of PAPR factor.
\end{abstract}

Keywords : BER, F-OFDM,OFDM, PSD.

\section{INTRODUCTION}

In the $4 \mathrm{G}$ networks OFDM is the dominant technology, to boost the spectrum efficiency [1] [2]. OFDM offers several advantages such as ease of implementation, robustness against multipath fading. OFDM signals require simple channel estimation techniques and can be used in MIMO transmission [3]. OFDM has number of drawbacks such as high PAPR, high OOBE and OFDM supports only one kind of waveform in the whole bandwidth. High PAPR problem in OFDM is mitigated using discrete Fourier transform based OFDM [4]. High OOBE in OFDM is owing to the use of rectangular pulse shape in time domain. These rectangular pulses produce sinc pulses in frequency domain. Large side-lobes of sinc pulses results in high OOBE. In order to overcome high OOBE problem filtered OFDM is proposed. Filtered OFDM uses sub-band filtering in which, complete bandwidth is divided into number of sub-bands and every sub-band is filtered so that different waveform parameter can be set according to the different service scenarios [5]. The filters in filtered OFDM are suitably designed to suppress the OOBE. The proposed F-OFDM has very low OOBE. While

Revised Manuscript Received on April 27, 2020.

* Correspondence Author

Asia Hazareena*, Department of ECE, P.A college of Engineering, Mangalore, India. Email: hazareenaut@gmail.com

Dr.B.Aziz Mustafa, Department of CS, Bearys Institite of Technology, Mangalore, India. Email: azizmusthafa@gmail.com

(C) The Authors. Published by Blue Eyes Intelligence Engineering and Sciences Publication (BEIESP). This is an open access article under the CC BY-NC-ND license (http://creativecommons.org/licenses/by-nc-nd/4.0/) designing a wireless communication model we need to consider the channel characteristics with respect to the wireless environment [6]. In this paper we have analyzed the performance of F-OFDM and OFDM on AWGN channel, Rayleigh channel and Rician channel. This paper has been organized in to six sections. Section 1 gives a brief summary of why OFDM should be replaced with F-OFDM. Section 2 gives the summary of related work carried out on F-OFDM. Section 3 presents system model of F-OFDM. Filter design and different channel models are presented in section 4 and section 5 . Simulation results are discussed in section 6 and section 7 presents conclusion.

\section{RELATED WORK}

Filtering has already been considered as a method to reduce the side lobes in literature [7]. With different filter designs it is possible to reduce out of band emission and support asynchronous transmission. Based on the type of filtering, filtered based OFDM are divided in to two types as subcarrier filtering and sub-band filtering. Filter-bank-multi-carrier modulation (FBMC) uses sub carrier filtering in which each sub carriers are filtered to reduce OOBE [3]. BER performance of OFDM has been compared with FBMC for nakagami fading channel in [8]. Since subcarrier filtering is used in FBMC, it requires very long filters. Long frequency selective filters used in FBMC causes problems when used in practical systems [9]. Also for multiple input multiple output (MIMO) applications FBMC is not compatible [10]. To overcome the disadvantages of sub carrier filtering based waveforms, sub-band filtering based waveforms were proposed. Sub-band filtering based waveform universal-filtered-multi-carrier (UFMC) was proposed in [11]. UFMC uses filter only at the transmitter side, and demodulation at the receiver is achieved by over sampled discrete Fourier Transform [12][13]. Sub-band filtering uses filters for each sub-band, so the length of the filters impulse response is reduced. Filtered OFDM which uses sub band filtering was proposed in [5]. Filtered discrete Fourier transform spread OFDMA was proposed in [14], in which at the transmitter side spectrum shaping filter is used for the elimination of side lobe leakage and at the receiver bank of filters are used to avoid inter-user interference.

\section{SYSTEM MODEL}

The operating principle of the F-OFDM transceiver is illustrated in Figure 1. F-OFDM divides the complete bandwidth in to number of sub-bands. Different filters can be used to filter different sub-bands. The choice of the filter in case of F-OFDM is flexible.

\section{Published By:}

Blue Eyes Intelligence Engineering \& Sciences Publication

(C) Convriaht: All riahts reserved. 
Filters are designed to reduce OOBE. Length of the Cyclic Prefix (CP) used in F-OFDM is same as OFDM. Since CP is used in F-OFDM it uses less complex Channel equalization techniques. During transmission, input binary data is modulated with quadrature phase shift keying (QPSK) modulation, output of QPSK is passed through IFFT to generate OFDM symbol. CP is added to the output of IFFT to safe guard data against ISI. After adding CP OFDM symbols are passed through finite impulse response (FIR) digital filter in which different window function can be used. Filtered OFDM signals are transmitted through different channels such as additive white Gaussian channel (AWGN), Rayleigh channel, and Rician channel. Matched filter is used at the receiver, which is equivalent to the complex conjugate time reversed version of the filter which is used at the transmitter side. Reverse process has been carried at the receiving side to estimate the input sequence. The basic OFDM transmitted signal is represented as

$x(t)=\sum_{n=0}^{N-1} X_{n}[k] e^{j 2 \pi \Delta f t} \quad \mathrm{kT}<\mathrm{t}<(\mathrm{k}+1) \mathrm{T}$

Where $X_{n}$ is the signal carried by the $n^{\text {th }}$ subcarrier. $N$ represents the number of subcarriers in OFDM. Total of $\mathrm{N}$ parallel symbols will be transmitted during each OFDM period of time $\mathrm{T}$.

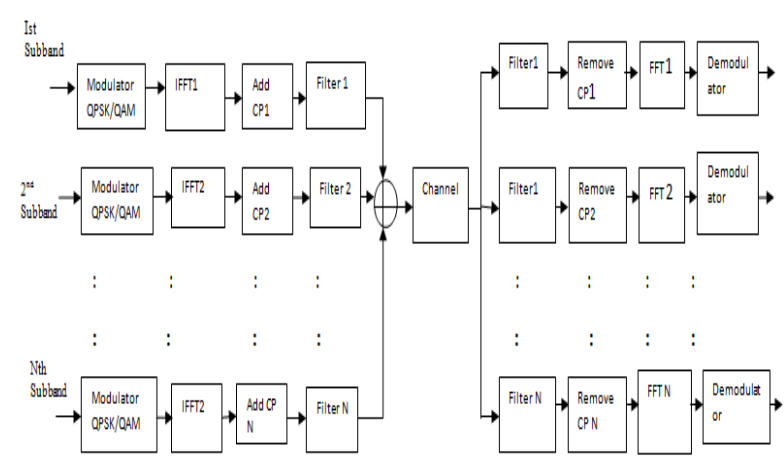

Figure 1. Block diagram of F-OFDM transmission

\section{FILTER DESIGN}

When the OFDM signal $\mathrm{x}(\mathrm{n})$ is passed through a filter we obtain filtered OFDM signal $s(n)$. $s(n)$ is the convolution between OFDM signal and filter function $\mathrm{f}(\mathrm{n})$.

$$
\mathrm{s}(\mathrm{n})=\mathrm{x}(\mathrm{n}) * \mathrm{f}(\mathrm{n})
$$

In frequency domain $S\left(e^{j \omega}\right)$ is obtained by multiplying the Fourier transform of $x(n)$ and $f(n)$

$$
\mathrm{S}\left(e^{j \omega}\right)=\mathrm{X}\left(e^{j \omega}\right) \mathrm{F}\left(e^{j \omega}\right)
$$

Filter is designed such that sufficient balance is provided between frequency and time localization. Consider a prototype filter with sinc impulse response h(n). Time windowing function $w(n)$ is applied on the impulse response of $\mathrm{h}(\mathrm{n})$ as

$$
\mathrm{f}(\mathrm{n})=\mathrm{h}(\mathrm{n}) \cdot \mathrm{w}(\mathrm{n})
$$

Windowing provides sufficient localization in time in the truncated filters impulse response, so induced inter symbol interference in the F-OFDM signal is reduced. We use different window functions such as Bartlett, Hamming, Hanning and Blackman.

\section{CHANNEL MODEL}

This section describes numerous impairments of practical wireless channel models. Basically there are three main basic wireless channel models i) Additive White Gaussian noise ii) Rayleigh iii) Rician.

\section{A. Additive white Gaussian noise (AWGN)}

Basic communication channel model is the AWGN standard channel model. When the signal is passed through the AWGN channel white Gaussian noise is added to the signal. Transmitted signal gets disturbed by the additive white Gaussian noise process.

Received signal is

$$
r(t)=s(t)+w(t)
$$

Where $\mathrm{s}(\mathrm{t})$ is the transmitted signal. $\mathrm{w}(\mathrm{t})$ is the additive white Gaussian noise.

\section{B. Rayleigh fading}

When there is no line of sight propagation between transmitter and receiver Rayleigh fading is applicable. In Rayleigh channel the parameters of the channels are due to multipath. Only reflected paths are available in Rayleigh fading. Probability density function (pdf) of Rayleigh fading distribution is

$$
p(l)=\frac{1}{\sigma^{2}} e^{\frac{-1^{2}}{2 \sigma^{2}}} \quad 0 \leq l \leq \infty
$$

\section{Rician fading}

In Rayleigh fading model it is assumed that all the reflections have a mean value of zero. This cannot be considered if there is line of sight path between transmitter and receiver. Rician distribution is used if there is a strong line of sight path along with reflected paths. Rician distribution is given by

$$
p(l)=\frac{l}{\sigma^{2}} e^{\frac{-\left(\mathbb{C}^{2}+A^{2}\right)}{\sigma^{2}}} I_{0}\left(\frac{A l}{\sigma^{2}}\right) \quad l \geq 0, \quad A \geq 0
$$

Where A denotes the peak amplitude of the line of sight component. $\mathrm{I}_{0}$ is the modified Bessel's function is of $0^{\text {th }}$ order of first kind.

\section{SIMULATION RESULT}

This section gives the performance evaluation of OFDM and F-OFDM. OFDM symbol duration 66.67 $\mu$ s is chosen according to LTE specifications, so subcarrier spacing is equal to $15 \mathrm{KHz}$. In F-OFDM 12 Resource blocks consisting of 50 subcarriers each are chosen. For the comparison, 600 OFDM subcarriers are chosen.

\section{A. PSD Comparison}

In Figure 2 PSD of F-OFDM is compared with conventional OFDM. F-OFDM has very low OOBE compared to OFDM. Figure 3 shows the PSD of F-OFDM with different window functions such as Bartlett, Hamming, Hanning and Blackman windows. It is clear that when Bartlett window is used OOBE performance is better than OFDM, with Blackman window it is possible to achieve very low OOBE.

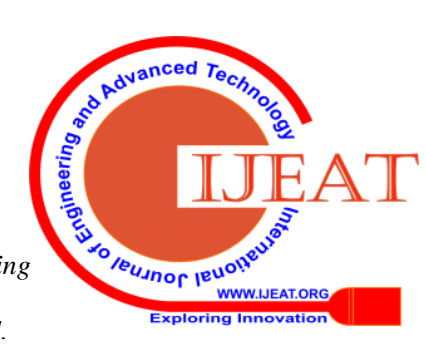




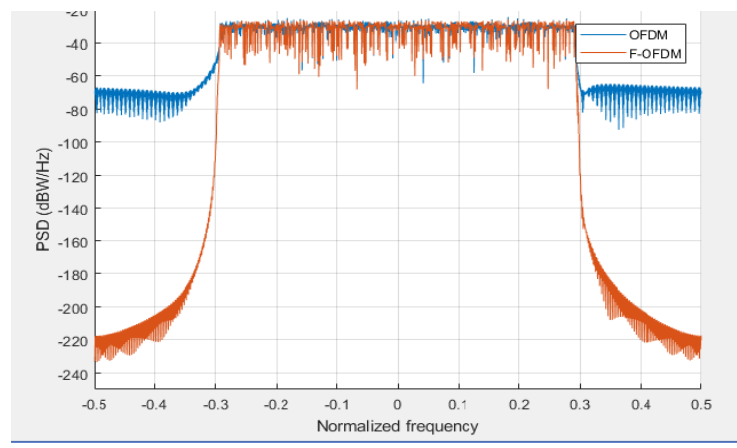

Figure 2. PSD's of OFDM and F-OFDM

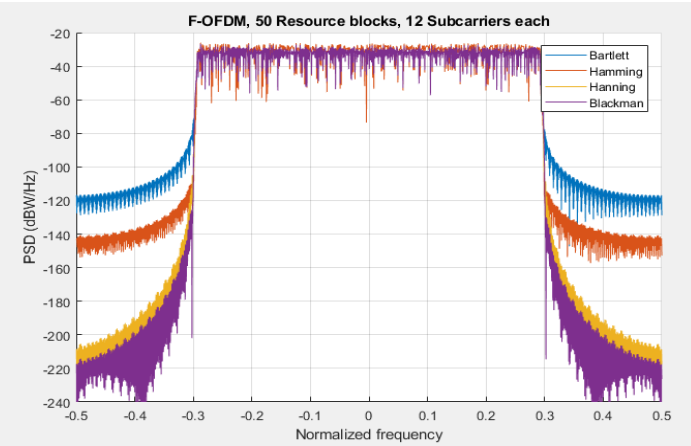

Figure 3. PSD's of F-OFDM for Bartlett, Hamming, Hanning and Blackman windows.

\section{B. BER Comparison}

F-OFDM not only has good OOBE performance, but also the BER performance of F-OFDM is same as conventional OFDM in case of AWGN Rayleigh and Rician channel. Figure 4 shows the BER performance of OFDM and F-OFDM when transmitted through AWGN channel. Figure 5 and Figure 6 gives BER comparison of F-OFDM and OFDM under Rayleigh fading scenario and Rician fading scenario.

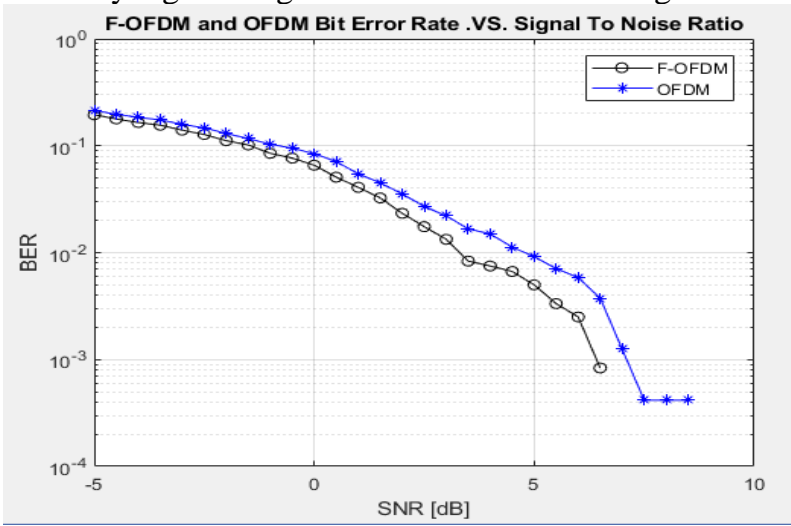

Figure 4 BER versus SNR comparison of OFDM and F-OFDM over AWGN channel.

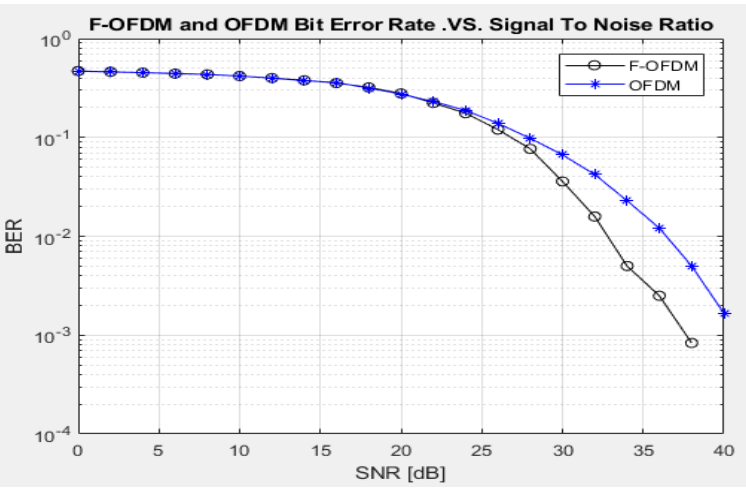

Figure 5. SNR versus BER comparision of OFDM and F-OFDM over Rayleigh channel.

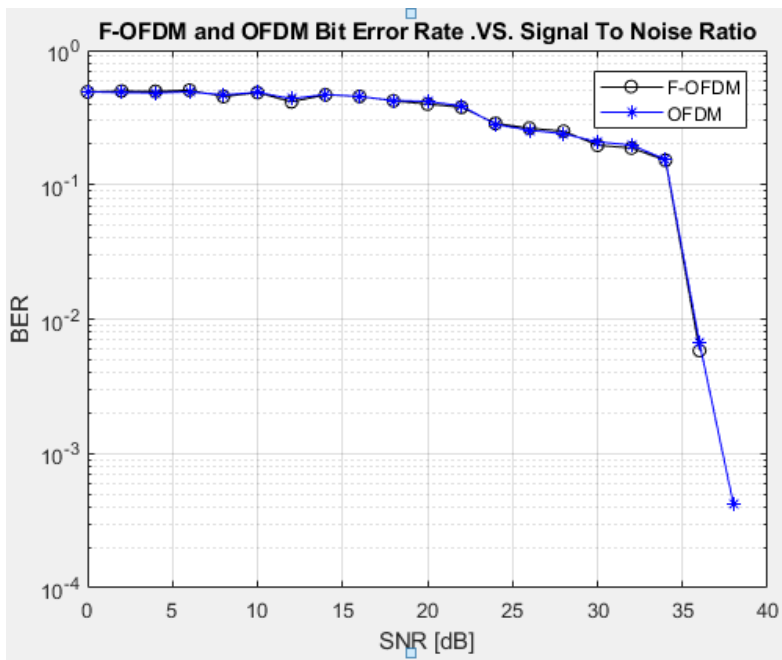

Figure 6. SNR versus BER comparision of OFDM and F-OFDM over Rician channel.

\section{PAPR Comparison}

The value of the transmitted signal PAPR for both OFDM and F-OFDM systems is given as

$$
\operatorname{PAPR}(\mathrm{s}(\mathrm{t}))_{\mathrm{dB}}=10 \log _{10}\left(\frac{\max \left(|s(t)|^{2}\right)}{\mid \overline{s(t) \|^{2}}}\right)
$$

Where $|\overline{S(t)}|$ is the transmitted signal's mean magnitude. PAPR of OFDM is $7.9283 \mathrm{~dB}$.

Table 1. PAPR of F-OFDM for different window functions

\begin{tabular}{|c|r|l|}
\hline Window Function & F-OFDM & \\
\hline Bartlett & $9.5965 \mathrm{~dB}$ & \\
Hanning & $9.6041 \mathrm{~dB}$ & \\
Hamming & $9.6025 \mathrm{~dB}$ & \\
Blackman & $9.6082 \mathrm{~dB}$ & \\
\end{tabular}

Table above shows the Complementary Distributive functions (CCDF) as a function of PAPR for F-OFDM when different window functions are used. PAPR increases when F-OFDM is used. Our result shows that F-OFDM with Blackman window can achieve much lower OOBE, but PAPR of F-OFDM with Blackman window is highest compared to F-OFDM with other window functions and OFDM.

\section{CONCLUSION}

Future 5G network requires asynchronous transmission, so filtered based OFDM has gained lots of attention. In this paper we have compared the performance of BER, PSD and PAPR factor of OFDM and F-OFDM. Compared to OFDM , F-OFDM can achieve much lower OOBE. Simulation result shows that BER performance of F-OFDM is almost same as OFDM in various channel environments such as AWGN, Rayleigh fading and Rician fading. For the cases considered it can be observed that PAPR value of OFDM is less than F-OFDM. F-OFDM with PAPR reduction technique can be the future work.

Published By:

Blue Eyes Intelligence Engineering \& Sciences Publication

(C) Convriaht: All riahts reserved.

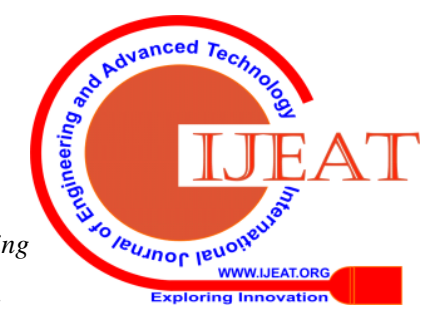




\section{REFERENCES}

1. Haitham J.Taha, M.F.Salleh, "Multi-carrier transmissiontechniques for wireless communication systems:A Survey,"WSEAS transactions on communications, vol. 8, 2009,pp. 457-470.

2. M. R. H Mondal, S. P. Majumder, "Analytical Performanceevaluation of space time coded MIMO OFDM systems impaired byfading and timing jitter" IEEE international conference oncomputer and information technology. 2009.

3. B. Farhang-Boroujney, "OFDM versus filter bank multicarrier" IEEE signal processing magazine, vol. 28, no.3,2011, pp. 92-112.

4. Xi zhang,Lei Chen,Jing Qiu and Jaad abdoli, "On the Waveformfor 5G.,” IEEE communication magazine, vol. 54, no.11,2016,pp. 74-80.

5. Xi Zhang, Ming Jia, Lei Chen, Jianglei Ma, Jing Qiu, "Filtered-OFDM - enabler for flexible waveform in the 5th generation cellular networks," in Proc. IEEE Global Commu.Conference (GLOBECOM), 2015.

6. A. Sudhir Babu, Dr. K.V Sambasiva Rao , "Evaluation of BER forAWGN, Rayleigh and Rician fading channels under variousmodulation Schemes," International Journal of computerapplication, vol. 26, no. 9, 2011, pp. 23-28.

7. Michael Faulkner, "The effect of filtering on the performance of OFDM systems," IEEE transactions of vehicular technology, vol. 49, no. 5, 2009, pp. 1877-1883.

8. Md. Motahar Hossain Mishu, M. Rubaiyat Hossain Mondal, "Effectiveness of filter bank multicarrier modulation for 5Gwireless communication," IEEE International Conference on advances in Electrical Engineering (ICAEE) 2017.

9. Y. Liu, X. Chen, Z. Zhong, "Waveform candidates for 5GNetworks: Analysis and Comparison,” IEEE Transl. J. Magn.Jpn., vol. 2, Aug. 1987, arXiv.org. pp. 1-17

10. M. Van Eeckhaute, A. Bourdoux, P. De Doncker, and F. Horlin, "Performance of emerging multicarrier waveforms for $5 \mathrm{G}$ asynchronous communications. EURASIP J. Wireless Commun.Networking 2017.

11. X.Wang, T. Wild, F. Schainch, A.Santos, "Universal filteredmulticarrier with leakage based filter optimization," In Proc. IEEE GLOBECOM Broadband Wireless Access Workshop,pp. 223-228.

12. Schaich, F. and T. Wild, "Waveform contender for 5G OFDM vs. FBMC vs. UFMC" International symposium on communications, control and signal processing 2013, pp. 123-135.

13. T. Wild, F. Schaich, Y. Chen, " $5 \mathrm{G}$ air interface design based onuniversal filtered OFDM," Int. Conf. Dig. Sig. Proc. (DSP) pp. 699-704.

14. Javad Abdoli, Ming Jia, Jianglei Ma, "Filtered OFDM: A newwaveform for future Wireless Systems," In Proc. IEEE 16th Int.Workshop Signal Process. Adv. Wireless Commu. (SPAWC),2015, pp. 66-70.

\section{AUTHORS PROFILE}

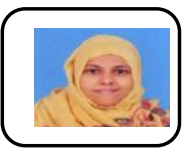

Asia Hazareena received her B.E degree in Electronics and Communication from Visvesvaraya Technological University in the year 2004 and M.Tech degree in Communication Engineering from National Institute of Technology Karnataka in the year 2010. She is currently persuing Ph.D at Visvesvaraya Technological University, Belagavi since 2014. Her research interests include Wireless Communication , Signal Processing etc.

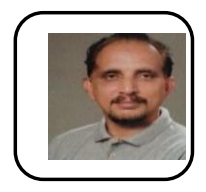

Aziz Musthafa B received his B.E degree in Electronics and Communication from Gulbarga University in the year 1990. M.S degree in Information Technology from Kuvempu university in the year 2006. He received his doctorate degree from Mangalore university in 2013. He has published papers in national and international conference and journals. His research interests include computer communication networks, Adhoc wireless networks, Security and cryptography etc. 